\title{
Simultaneous Reconstructions of Absorption Density and Wave Speed with Photoacoustic Measurements *
}

\author{
Andreas Kirsch ${ }^{\dagger} \quad$ Otmar Scherzer ${ }^{\ddagger}$
}

October 24, 2018

\begin{abstract}
In this paper we propose an approach for simultaneous identification of the absorption density and the speed of sound by photoacoustic measurements. Experimentally our approach can be realized with sliced photoacoustic experiments. The mathematical model for such an experiment is developed and exact reconstruction formulas for both parameters are presented.
\end{abstract}

\section{Introduction}

In this paper we propose an approach for simultaneous identification of the absorption density and the speed of sound by photoacoustic measurements.

In standard photoacoustic experiments the object is uniformly illuminated by a short electromagnetic impulse. Recently sectional photoacoustic imaging techniques [17, 22, 9, 10] and according reconstructions techniques [4] have been developed. In sectional experiments thin hyperbola shaped like regions of the specimen are imaged (see Figure 11) sequentially. Experimentally, one can perform sectional photoacoustic imaging by focused illumination combined with focusing detectors. The focused illumination is achieved by using cylindrical lenses in front of the object. Moreover, contemporary focusing ultrasonic detectors have a spherical or cylindrical shape, thus the detector surface plays the role of the acoustic lens. The generated ultrasonic wave is refracted by a suitable acoustic lens such that out-of-plane (center of the hyperbola shaped regions) signals are generally weak and can be neglected. Thus essentially only signals emerging from the imaging plane are collected at the detector. This justifies

\footnotetext{
*The work has been supported by the Austrian Science Fund (FWF) within the national research network Photoacoustic Imaging in Biology and Medicine, project S10505-N20.

${ }^{\dagger}$ Karlsruhe Institute of Technology, Department of Mathematics, Kaiserstraße 89, 76128 Karlsruhe, Germany

$¥$ Computational Science Center, University of Vienna, Nordbergstraße 15, A-1090 Vienna, Austria, and Johann Radon Institute for Computational and Applied Mathematics (RICAM), Austrian Academy of Sciences, Altenbergerstraße 69, A-4040 Linz, Austria (otmar.scherzer@univie.ac.at).
} 
that the illumination can be assumed restricted to a single plane (line in 2D). However, such a model requires a low scattering coefficient of the sample (which model organism specimens like the Zebra fish have). Opposed to sectional $3 D$

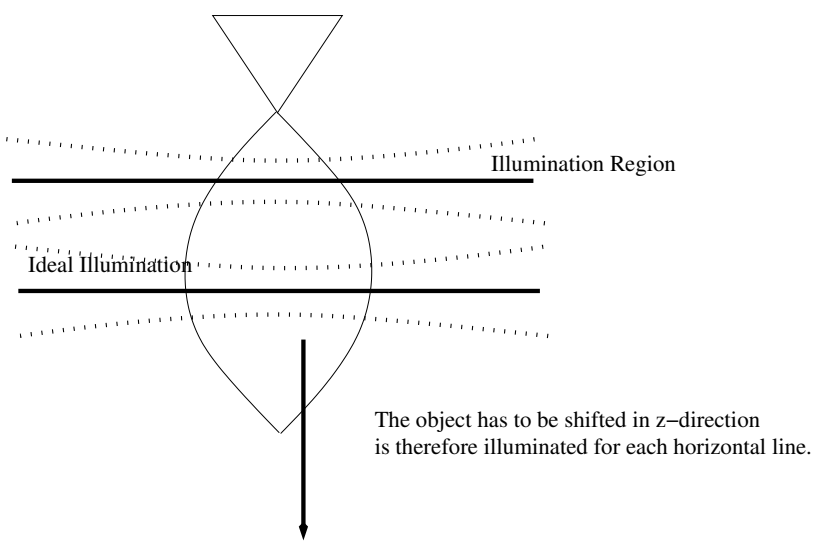

Fig. 1: Conventional sectional photoacoustic imaging.

photoacoustic imaging, where stacks of complementary two-dimensional projection images are produced, the proposed approach for simultaneous imaging consists in performing overlapping sliced imaging by rotation and translation of the specimen. This, also generates enough data for reconstructing the two independent parameter functions, speed of sound and absorption density, (see Figure 22. By now the approach presented here is by far from being experi-

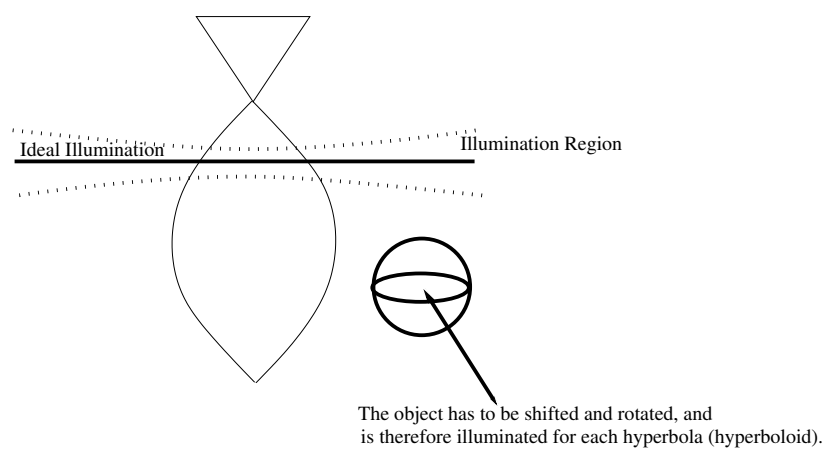

Fig. 2: Sectional imaging in all directions produces enough data to reconstruct both imaging functions.

mentally economical in the sense that a quick dimension analysis shows that we acquire much too many data - in $\mathbb{R}^{3}$ we reconstruct two 3-dimensional functions from eventually six dimensional data. It is the goal of this paper, however, not to present the most economical approach, but to show that it is possible to derive exact reconstruction formulas for both imaging parameter functions.

The literature on reconstruction formulas and back-projection algorithms for photoacoustic imaging is vast. Wang et al. developed reconstruction formulas for cylindrical, spherical, and planar measurement geometries in a series of papers $27,26,25,28$, and recently many more algorithms based on reconstruction 
formulas have been developed (see the survey [15). As it becomes transparent below we can make use of reconstruction formulas from photoacousticsessentially we make extensive use of inversion formulas for the spherical mean operator. Exact reconstruction formulas for the wavespeed function in ultrasound reflectivity tomography are based on the Born approximation to the wave equation, which is valid for moderately varying speed of sound. Reconstruction formulas for ultrasound reflectivity tomography have already been derived by Norton \& Linzer [18, 19] and are also based on inversion formulas for the spherical mean operator. The possibility of exact inversion in both fields supports to derive exact inversion formulas for both parameters.

The proposed method is a hybrid and quantitative imaging methods (see 3, 14, 2]) for some recent surveys. Most closely related to our approach is the work of Stefanov and Uhlmann 24] which presented a photoacoustic experiment for recovering either the sound speed or absorption density. A substitute to our work is also the recovery of the absorption density function for inhomogeneous wave speed [1, 11, 23].

The reconstruction formulas for simultaneous imaging utilize techniques from reflectivity imaging and photoacoustic imaging. In the current state of research we can provide exact reconstruction formulas, but when we try to be economical, i.e., using less slicing experiments, we would require reconstruction formulas, which have not been developed so far. This is highlighted in Section 2, The outline of this paper is as follows: In Section 2 we discuss the mathematical model and discuss the non-economicality of proposed model, which leaves room for further improvement of the results. In Section 3 we transform the model into the Fourier domain, from which in Sections 3.2, 3.1 exact reconstruction formulas are derived in 2D, 3D, respectively. The Appendix provides the exact definitions used in this paper.

\section{Model}

In $\mathbb{R}^{n}$, we consider the following Cauchy problem for the wave equation:

$$
\begin{aligned}
\frac{1}{c^{2}} \partial_{t t} \tilde{u}-\Delta \tilde{u} & =0 \quad \text { in } \mathbb{R}^{n} \times \mathbb{R}_{>0}, \\
\tilde{u}(x, 0) & =f(x) \delta_{r, \theta}(x) \quad \text { in } \mathbb{R}^{n}, \\
\partial_{t} \tilde{u}(x, 0) & =0 \text { in } \mathbb{R}^{n} .
\end{aligned}
$$

where $c=c(x)$ denotes the speed of sound and $\delta_{r, \theta}(x)=\delta(\operatorname{dist}(x, E(r, \theta)))$ where $E(r, \theta)$ is the $(n-1)$-dimensional hyperplane with distance $r$ from the origin and orientation $\theta \in S^{n-1}$. This is the photoacoustic equation with sliced illumination in the plane $E(r, \theta)$. It is the ultimate aim to reconstruct $c$ and $f$ from measurements of $\tilde{u}$ on some hypersurface $\Gamma$ (see below).

We consider the Born approximation; that is, we expand $\tilde{u}$ formally with respect to the contrast function $q:=1 / c^{2}-1$ and consider only terms of order at most $q$. This leads to the decomposition $\tilde{u} \approx u+v$ where $u=u^{r, \theta}$ is the solution of 
the wave equation

$$
\begin{aligned}
\partial_{t t} u-\Delta u & =0 \quad \text { in } \mathbb{R}^{n} \times \mathbb{R}_{>0}, \\
u(x, 0) & =f(x) \delta_{r, \theta}(x) \quad \text { in } \mathbb{R}^{n}, \\
\partial_{t} u(x, 0) & =0 \quad \text { in } \mathbb{R}^{n},
\end{aligned}
$$

and $v=v^{r, \theta}$ solves

$$
\begin{aligned}
\partial_{t t} v-\Delta v & =-q(x) \partial_{t t} u \quad \text { in } \mathbb{R}^{n} \times \mathbb{R}_{>0} \\
v(x, 0) & =0 \quad \text { in } \mathbb{R}^{n} \\
\partial_{t} v(x, 0) & =0 \quad \text { in } \mathbb{R}^{n} .
\end{aligned}
$$

The dependence of $v$ on $r, \theta$ is through the source function $u$.

It is our goal to reconstruct $q$ and $f$ from measurements of

$$
m^{r, \theta}(x, t)=u^{r, \theta}(x, t)+v^{r, \theta}(x, t), \quad(x, t) \in \Gamma \times(0, T),
$$

where $\Gamma$ is a $(n-1)$-dimensional hypersurface in $\mathbb{R}^{n}$. Currently everything is fixed to complete measurements on the whole surface $\Gamma$.

We make the following assumptions:

1. $\Gamma=\partial B$ for some open and connected set $B \subset \mathbb{R}^{n}$ with smooth boundary. As typically in photoacoustics, we consider $B$ to be a sphere, a circle, or a halfplane.

2. $f$ is the sum of some known initial distribution $f_{0}$ with compact support and some unknown term $f_{1}$ to be determined.

3. The supports of $f_{1}$ and $q$ are both contained in $\Omega$ for some bounded domain $\Omega \subset B$,

4. $f_{0}, f_{1}$ and $q$ are smooth and $f(x)=f_{0}(x)+f_{1}(x) \neq 0$ for all $x \in \bar{B}$.

\section{Dimensionality Analysis}

In the $n$-dimensional setting, we record measurements (cf. (2.1)) for every $(x, t) \in \Gamma \times(0, T)$ and every $(r, \theta) \in(0, \infty) \times S^{n-1}$. That is, the recorded data are $2 n$-dimensional. The data to be recovered are $f_{1}$ and $q$, which are two $n$-dimensional functions. In general we think that we record too many data for the purpose of reconstructing $f_{1}$ and $q$-however, since we rely on Radon transforms techniques for exact inversion, we accept this disadvantage for the mathematical studies.

\section{$3 \quad$ Fourier Reconstruction Formulas}

In this section we derive exact reconstruction formulas for $f_{1}$ and $g$. First we derive a general inversion formula, which is then evaluated differently in $2 \mathrm{D}$ and $3 \mathrm{D}$. 


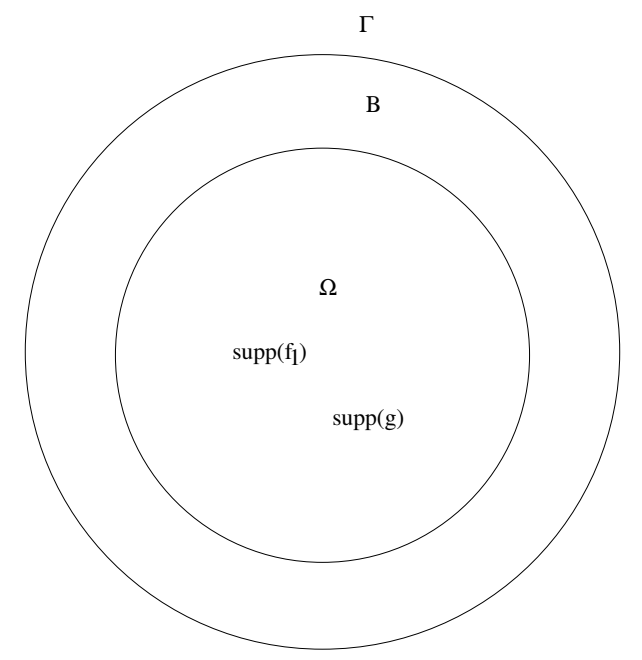

Fig. 3: Schematic representation of the domains and supports.

In the following we omit the superscripts $(r, \theta)$ for the sake of convenience of notation. Let

$$
\hat{u}(x, k)=\mathcal{F} u(x, k)=\int_{0}^{\infty} u(x, t) e^{\mathrm{i} k t} d t, \quad x \in \mathbb{R}^{n}, k \in \mathbb{R},
$$

be the Fourier transform - note that $u$ vanishes for $t<0$. We assume that $u$ is bounded in $\mathbb{R}^{n} \times \mathbb{R}_{>0}$ such that $u(x, \cdot) \in L^{2}(0, \infty)$ for every $x \in \mathbb{R}^{n}$. Then we note that $\hat{u}$ has the following properties (by the theorem of Paley-Wiener):

(a) $u(x, \cdot)$ has a holomorphic extension into $\mathbb{C}_{+}:=\{z \in \mathbb{C}: \Im z>0\}$ for all $x \in \mathbb{R}^{n}$,

(b) for every $x \in \mathbb{R}^{n}$ it holds that $\int_{-\infty}^{\infty}\left|\hat{u}\left(x, k_{1}+i k_{2}\right)-\hat{u}\left(x, k_{1}\right)\right|^{2} d k_{1} \longrightarrow 0$ as $k_{2}$ tends to zero,

(c) $\hat{u}(\cdot, k)$ is bounded in $\mathbb{R}^{n}$ for all $k \in \mathbb{C}_{+}$.

We have

$$
\begin{aligned}
\hat{u}(x, k) & =\mathcal{F} u(x, k)=\int_{0}^{\infty} u(x, t) e^{\mathrm{i} k t} d t \\
& =\frac{1}{\mathrm{i} k}\left[\left.u(x, t) e^{\mathrm{i} k t}\right|_{t=0} ^{\infty}-\int_{0}^{\infty} \partial_{t} u(x, t) e^{\mathrm{i} k t} d t\right] \\
& =-\frac{1}{\mathrm{i} k} u(x, 0)-\frac{1}{(\mathrm{i} k)^{2}}\left[\left.\partial_{t} u(x, t) e^{\mathrm{i} k t}\right|_{t=0} ^{\infty}-\int_{0}^{\infty} \partial_{t t} u(x, t) e^{\mathrm{i} k t} d t\right] \\
& =-\frac{1}{\mathrm{i} k} u(x, 0)-\frac{1}{k^{2}} \Delta \hat{u}(x, k),
\end{aligned}
$$

or in other words

$$
\Delta \hat{u}(x, k)+k^{2} \hat{u}(x, k)=\mathrm{i} k u(x, 0) .
$$


Moreover, we have

$$
\begin{aligned}
\hat{v}(x, k) & =\mathcal{F} v(x, k)=-\frac{1}{k^{2}} \int_{0}^{\infty} v_{t t}(x, t) e^{\mathrm{i} k t} d t \\
& =-\frac{1}{k^{2}} \int_{0}^{\infty}\left[\Delta v(x, t)-q(x) \partial_{t t} u(x, t)\right] d t \\
& =-\frac{1}{k^{2}} \Delta \hat{v}(x, k)+\frac{q(x)}{k^{2}} \int_{0}^{\infty} \partial_{t t} u(x, t) e^{\mathrm{i} k t} d t \\
& =-\frac{1}{k^{2}} \Delta \hat{v}(x, k)+\frac{q(x)}{k^{2}} \Delta \hat{u}(x, k),
\end{aligned}
$$

and thus

$$
\begin{aligned}
\Delta \hat{v}(x, k)+k^{2} \hat{v}(x, k) & =q(x) \Delta \hat{u}(x, k) \\
& =-k^{2} q(x) \hat{u}(x, k)+\mathrm{i} k q(x) u(x, 0) .
\end{aligned}
$$

Let $\Phi_{k}$ be the (radiating) fundamental solution of the Helmholtz equation $\Delta \hat{u}+$ $k^{2} \hat{u}=0$ in $\mathbb{R}^{n}$ for $k \in \mathbb{C}$ with $\Im z \geq 0$; that is, in particular for $n=2,3$,

$$
\Phi_{k}(x, y):=\left\{\begin{array}{cl}
\frac{\exp (i k|x-y|}{4 \pi|x-y|} & \text { for } n=3, \\
\frac{\mathrm{i}}{4} H_{0}^{(1)}(k|x-y|) & \text { for } n=2,
\end{array} \quad x \neq y,\right.
$$

where $H_{0}^{(1)}$ denotes the Hankel function of the first kind and order zero. Then one particular solution of (3.1) is given by

$$
\begin{aligned}
\hat{u}(x, k) & =-\mathrm{i} k \int_{\mathbb{R}^{n}} u(y, 0) \Phi_{k}(x, y) d y \\
& =-\mathrm{i} k \int_{y \in E(r, \theta)} f(y) \Phi_{k}(x, y) d s(y) .
\end{aligned}
$$

We note that this particular solution satisfies the properties (a), (b), (c) from the beginning of this section. Any other solution is of the form $\hat{u}(\cdot, k)+w(\cdot, k)$ where $w$ satisfies $\Delta w+k^{2} w=0$ in $\mathbb{R}^{n}$. The requirement that the solution satisfies the properties (a), (b), (c) from the beginning of this section implies that $w$ vanishes. Indeed, for $k \in \mathbb{C}_{+}$the function $w(\cdot, k)$ has to be bounded by property (c), therefore its Fourier transform (in the distributional sense) with respect to $x \in \mathbb{R}^{n}$ satisfies

$$
\mathcal{F}_{x} w(y, k)\left[k^{2}-|y|^{2}\right]=0 \text { for all } y \in \mathbb{R}^{n}
$$

which implies $\mathcal{F}_{x} w(\cdot, k)=0$ because $k^{2}-|y|^{2}$ does not vanish for $k \in \mathbb{C}_{+}$. Therefore, also $w(\cdot, k)=0$ in $\mathbb{R}^{n}$ for all $k \in \mathbb{C}_{+}$and, by the continuity property (b), also $w(\cdot, k)=0$ in $\mathbb{R}^{n}$ for all $k \in \mathbb{C}$ with $\Im \geq 0$. 
We also have

$$
\begin{aligned}
& \hat{v}(x, k) \\
= & k^{2} \int_{\mathbb{R}^{n}} q(y) \hat{u}(y, t) \Phi_{k}(x, y) d y-\mathrm{i} k \int_{\mathbb{R}^{n}} q(y) f(y) \delta_{r, \theta}(y) \Phi_{k}(x, y) d y \\
= & -\mathrm{i} k^{3} \int_{\mathbb{R}^{n}} q(y) \int_{z \in E(r, \theta)} f(z) \Phi_{k}(y, z) d s(z) \Phi_{k}(x, y) d y \\
& \quad-\mathrm{i} k \int_{z \in E(r, \theta)} q(z) f(z) \Phi_{k}(y, z) d s(z) \\
= & -\mathrm{i} k \int_{z \in E(r, \theta)} f(z)\left[k^{2} \int_{\mathbb{R}^{n}} q(y) \Phi_{k}(y, z) \Phi_{k}(x, y) d y+q(z) \Phi_{k}(x, z)\right] d s(z) .
\end{aligned}
$$

In summary, we have

$$
\begin{aligned}
& \hat{m}^{r, \theta}(x, k)= \hat{u}^{r, \theta}(x, k)+\hat{v}^{r, \theta}(x, k)=\hat{u}(x, k)+\hat{v}(x, k) \\
&=-\mathrm{i} k \int_{z \in E(r, \theta)} f(z)\left[k^{2} \int_{\mathbb{R}^{n}} q(y) \Phi_{k}(y, z) \Phi_{k}(x, y) d y\right. \\
&\left.\quad+(q(z)+1) \Phi_{k}(x, z)\right] d s(z) \\
&= \\
& R[(f(\cdot) L(x, \cdot, k))](r, \theta),
\end{aligned}
$$

where $R[f](r, \theta)$ is the $(n-1)$-dimensional Radon transform of $f$ in direction $(r, \theta)$ and

$$
L(x, z, k)=(-\mathrm{i} k)^{3} \int_{\mathbb{R}^{n}} q(y) \Phi_{k}(y, z) \Phi_{k}(y, x) d y-\mathrm{i} k(q(z)+1) \Phi_{k}(x, z)
$$

for $x \in \Gamma, z \in B, k \in \mathbb{R}, x \neq z$.

In other words, we have

$$
\mathcal{F}^{-1}\left(R^{-1}(\hat{u}+\hat{v})\right)(x, z, t)=f(z) \check{L}(x, z, t)
$$

for $x \in \Gamma, z \in B, k \in \mathbb{R}, x \neq z$.

Therefore, from the knowledge of $\hat{m}^{r, \theta}(x, k)=\hat{u}(x, k)+\hat{v}(x, k)$ for all $x \in \Gamma$, $k \in \mathbb{R}, r \geq 0$, and $\theta \in S^{n-1}$ we can determine

$$
f(z) \check{L}(x, z, t)=\left(f_{0}(z)+f_{1}(z)\right) \check{L}(x, z, t)
$$

for all $x \in \Gamma, z \in B$ with $x \neq z$, and $t \geq 0$.

\subsection{D Domain}

For $n=3$ we recall that

$$
\Phi_{k}(x, y)=\frac{e^{\mathrm{i} k|x-y|}}{4 \pi|x-y|}, \quad x \neq y .
$$

Thus from 3.2 it follows that

$L(x, z, k)=(-\mathrm{i} k)^{3} \frac{1}{16 \pi^{2}} \int_{\mathbb{R}^{3}} q(y) \frac{e^{\mathrm{i} k(|y-z|+|x-y|)}}{|x-y||y-z|} d y-\mathrm{i} k(q(z)+1) \frac{e^{\mathrm{i} k|x-z|}}{4 \pi|x-z|}$. 
Taking the inverse Fourier transform with respect to $k$ gives

$$
\begin{aligned}
\check{L}(x, z, t)= & \frac{1}{32 \pi^{3}} \partial_{t t t}\left(\int_{\mathbb{R}^{3}} \frac{q(y)}{|x-y||y-z|} \int_{-\infty}^{\infty} e^{\mathrm{i} k(|y-z|+|x-y|-t)} d k d y\right) \\
& +(q(z)+1) \frac{1}{8 \pi^{2}|x-z|} \partial_{t}\left(\int_{-\infty}^{\infty} e^{\mathrm{i} k(|x-z|-t)} d k\right) \\
= & \frac{1}{32 \pi^{3}} \partial_{t t t}\left(\int_{\mathbb{R}^{3}} \delta(|y-z|+|y-x|-t) \frac{q(y)}{|z-y||y-x|} d s(y)\right) \\
& -\frac{q(z)+1}{8 \pi^{2}|x-z|} \delta^{\prime}(|x-z|-t) .
\end{aligned}
$$

Twice Integration with respect to $t$ gives

$$
\begin{aligned}
\int_{0}^{s_{2}} \int_{0}^{s_{1}} \check{L}(x, z, \tau) d \tau d s_{1} \\
=\frac{q(z)+1}{8 \pi^{2}|x-z|} H\left(s_{2}-|x-z|\right) \\
\quad+\partial_{s_{2}}(\underbrace{\int_{\mathbb{R}^{3}} \delta\left(|y-z|+|y-x|-s_{2}\right) \frac{q(y)}{32 \pi^{3}|z-y||y-x|} d s(y)}_{=: \mathcal{N}\left(x, z, s_{2}\right)}),
\end{aligned}
$$

where we used the Heavyside function $H(\tau)=1$ for $\tau>0$ and $H(\tau)=0$ for $\tau<0$. The initial conditions for the second term vanish since for $t=0$ the domain of integration (w.r.t. $y$ ) is empty.

Integrating once more, we get

$$
\begin{aligned}
\Psi(x, z, t) & :=\int_{0}^{t} \int_{0}^{s_{2}} \int_{0}^{s_{1}} \check{L}(x, z, \tau) d \tau d s_{1} d s_{2} \\
& =\frac{q(z)+1}{8 \pi^{2}|x-z|} \int_{0}^{t} H\left(s_{2}-|x-z|\right) d s_{2}+\mathcal{N}[q](x, z, t) .
\end{aligned}
$$

Now, we note that

$$
\int_{0}^{t} H\left(s_{2}-|x-z|\right) d s_{2}=(t-|x-z|)^{+}:=(t-|x-z|) H(t-|x-z|),
$$

and thus

$$
\Psi(x, z, t)=\frac{q(z)+1}{8 \pi^{2}|x-z|}(t-|x-z|)^{+}+\mathcal{N}[q](x, z, t) .
$$

Both terms vanish for $t<|x-z|$.

We recall that $M(x, z, t):=f(z) \Psi(x, z, t)$ is known from measurement data for all $x \in \Gamma, z \in B$, and $t>0$. First we take $z \in B \backslash \Omega$. Then $q(z)=0$ and $f(z)=f_{0}(z)$, thus $\Psi(x, z, t)=M(x, z, t) / f_{0}(z)$ is known and

$$
\frac{M(x, z, t)}{f_{0}(z)}-\frac{(t-|x-z|)^{+}}{8 \pi^{2}|x-z|}=\mathcal{N}[q](x, z, t) .
$$


Again, the left hand side is known. Now we let $z$ tend to $x$. The limit on the right hand side exists, thus also on the left hand side, and therefore for all $x \in \Gamma$ and $t>0$ we have

$$
\begin{aligned}
\lim _{z \rightarrow x}\left[\frac{M(x, z, t)}{f_{0}(z)}-\frac{t}{8 \pi^{2}|x-z|}\right] & =\frac{1}{8 \pi^{3} t^{2}} \int_{\mathbb{R}^{3}} \delta(|y-x|-t / 2) q(y) d s(y) \\
& =\frac{1}{8 \pi} \mathcal{M}_{2}[q](x, t / 2),
\end{aligned}
$$

where $\mathcal{M}_{2}[q]$ is the spherical mean operator (see Appendix).

Thus the reconstruction algorithm is as follows:

1. Calculate the product $f(z) \check{L}(x, z, t)$ from 3.3 for all $x \in \Gamma$, $z \in B$ and $t>0$. By integration (see (3.4)), this yields the knowledge of $M(x, z, t):=f(z) \Psi(x, z, t)$ for all $x \in \Gamma, z \in B$ and $t>0$.

2. Solve (3.6) for $q$ in $\Omega$ by inverting the spherical mean operator.

3. Compute $\Psi(x, z, t)$ for all $x \in \Gamma, z \in \Omega$ and $t>0$ from (3.5).

4. Finally, compute $f$ from $f(z)=M(x, z, t) / \Psi(x, z, t)$ for $z \in \Omega$.

Remark 3.1 The operator $\mathcal{N}[q](x, z, t)$ is the rotational ellipsoidal mean operator with focal points $x$ and $z$. Thus the integral equation (3.5); that is,

$$
\Psi(x, z, t)=\frac{q(z)+1}{8 \pi^{2}|x-z|}(t-|x-z|)^{+}+\mathcal{N}[q](x, z, t),
$$

can, for instance, be considered as a fixed point equation for $q$ involving the ellipsoidal mean operator and can be solved by the fixed point iteration

$$
\Psi(x, z, t)=\frac{q_{n}(z)+1}{8 \pi^{2}|x-z|}(t-|x-z|)^{+}+\mathcal{N}\left[q_{n-1}\right](x, z, t), n=1,2, \ldots
$$

Ellipsoidal mean operators have been studied in John's book [12].

\subsection{D Domain}

In $\mathbb{R}^{2}$ we recall that the fundamental solution is given by

$$
\Phi_{k}(x, y)=\frac{\mathrm{i}}{4} H_{0}^{(1)}(k|x-y|), \quad x \neq y .
$$

We have to compute the inverse Fourier transform of the product $\Phi_{k}(x, y) \Phi_{k}(z, y)$ (see (3.2) and use the convolution theorem. First we have that

$$
\frac{i}{4} \mathcal{F}^{-1}\left(H_{0}^{(1)}(\cdot|x-y|)\right)(t)=\left\{\begin{array}{cl}
\frac{1}{2 \pi \sqrt{t^{2}-|x-y|^{2}},} & t>|x-y|, \\
0, & t<|x-y| .
\end{array}\right.
$$


The convolution for the inverse Fourier transform is given by the formula $\mathcal{F}^{-1}(f g)=\check{f} * \check{g}$; that is,

$$
\mathcal{F}^{-1}(f g)(t)=(\check{f} * \check{g})(t)=\int_{-\infty}^{\infty} \check{f}(\tau) \check{g}(t-\tau) d \tau .
$$

Therefore,

$$
\begin{aligned}
& \left(\frac{i}{4}\right)^{2} \mathcal{F}^{-1}\left(H_{0}^{(1)}(\cdot|x-y|) H_{0}^{(1)}(\cdot|z-y|)\right)(t)
\end{aligned}
$$

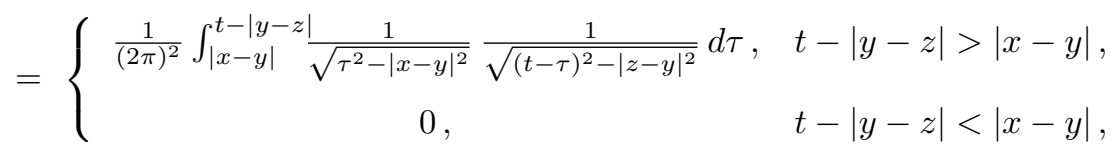

and thus from 3.2

$$
\begin{aligned}
& \check{L}(x, z, t) \\
& =\frac{1}{4 \pi^{2}} \partial_{t t t}\left(\int_{\mathbb{R}^{2}} q(y) \int_{|x-y|}^{t-|y-z|} \frac{1}{\sqrt{\tau^{2}-|x-y|^{2}}} \frac{1}{\sqrt{(t-\tau)^{2}-|z-y|^{2}}} d \tau d y\right) \\
& \quad+\frac{1}{2 \pi} \partial_{t}\left(\frac{q(z)+1}{\sqrt{t^{2}-|x-z|^{2}}} H(t-|x-z|)\right),
\end{aligned}
$$

for $t-|y-z|>|x-y|$ where $H$ denotes again the Heaviside function. Again, as in $3 \mathrm{D}$, both terms vanish for $t<|x-z|$.

Letting $z$ tend to $x$ (thus $t>|x-z|$ for $z$ sufficiently close to $x$ ) yields

$$
\begin{aligned}
& \check{L}(x, x, t) \\
& =\frac{1}{4 \pi^{2}} \partial_{t t t}\left(\int_{2|y-x|<t} q(y) \int_{|x-y|}^{t-|y-z|} \frac{1}{\sqrt{\tau^{2}-|x-y|^{2}}} \frac{1}{\sqrt{(t-\tau)^{2}-|x-y|^{2}}} d \tau d y\right) \\
& \quad+\frac{1}{2 \pi} \partial_{t}\left(\frac{1}{t}\right) .
\end{aligned}
$$

Note that $q(x)$ vanishes for $x \in \Gamma$. Using polar coordinates and denoting

$$
k(t, r):=\left\{\begin{array}{cc}
\int_{r}^{t-r} \frac{1}{\sqrt{\tau^{2}-r^{2}}} \frac{1}{\sqrt{(t-\tau)^{2}-r^{2}}} d \tau, & t>2 r>0, \\
0, & 0<t<2 r,
\end{array}\right.
$$

we therefore get

$$
\int_{0}^{t / 2} k(t, r) r \int_{0}^{2 \pi} q\left(x+r\left(\begin{array}{c}
\cos \rho \\
\sin \rho
\end{array}\right)\right) d \rho d r=2 \pi \int_{0}^{t / 2} \mathcal{M}_{1}[q](x, r) k(t, r) r d r
$$

with the one dimensional circular mean operator $\mathcal{M}_{1}$. Using the notations

$$
\begin{aligned}
\tilde{k}(t, r) & :=r k(2 t, r) \text { and } \\
\Phi(x, t) & :=4 \pi^{2} \int_{0}^{t} \int_{0}^{s_{2}} \int_{0}^{s_{1}} \check{L}(x, x, \tau) d \tau d s_{1} d s_{2}-2 \pi(t \log (t)-t),
\end{aligned}
$$


we get, for fixed $x$, the Volterra integral equation for $\mathcal{M}_{1}[q](x, \cdot)$ :

$$
\Phi(x, t)=\int_{0}^{t} \tilde{k}(t, r) \mathcal{M}_{1}[q](x, r) d r, \quad t \in(0, T] .
$$

We express $k$ as a complete elliptic integral: We introduce the variable $\phi \in$ $(-\pi / 2, \pi / 2]$ by $\tau=\frac{t}{2}+\left(\frac{t}{2}-r\right) \sin \phi$. Then $d \tau=\left(\frac{t}{2}-r\right) \cos \phi d \phi$ and, with $t^{\prime}=t / 2$,

$$
\begin{aligned}
\tau^{2}-r^{2} & =(\tau-r)(\tau+r) \\
& =\left[\left(t^{\prime}-r\right)+\left(t^{\prime}-r\right) \sin \phi\right]\left[\left(t^{\prime}+r\right)+\left(t^{\prime}-r\right) \sin \phi\right], \\
(t-\tau)^{2}-r^{2} & =(t-\tau-r)(t-\tau+r) \\
& =\left[\left(t^{\prime}-r\right)-\left(t^{\prime}-r\right) \sin \phi\right]\left[\left(t^{\prime}+r\right)-\left(t^{\prime}-r\right) \sin \phi\right] .
\end{aligned}
$$

For the product we conclude that

$$
\begin{aligned}
& {\left[\tau^{2}-r^{2}\right]\left[(t-\tau)^{2}-r^{2}\right]} \\
& \quad=\left[\left(t^{\prime}-r\right)^{2}-\left(t^{\prime}-r\right)^{2} \sin ^{2} \phi\right]\left[\left(t^{\prime}+r\right)^{2}-\left(t^{\prime}-r\right)^{2} \sin ^{2} \phi\right] \\
& \quad=\left(t^{\prime}-r\right)^{2} \cos ^{2} \phi\left[\left(t^{\prime}+r\right)^{2}-\left(t^{\prime}-r\right)^{2} \sin ^{2} \phi\right] .
\end{aligned}
$$

Therefore,

$$
\begin{aligned}
k(t, r) & =\int_{-\pi / 2}^{\pi / 2} \frac{d \phi}{\sqrt{\left(t^{\prime}+r\right)^{2}-\left(t^{\prime}-r\right)^{2} \sin ^{2} \phi}} \\
& =\frac{2}{t^{\prime}+r} \int_{0}^{\pi / 2} \frac{d \phi}{\sqrt{1-\left(\frac{t^{\prime}-r}{t^{\prime}+r}\right)^{2} \sin ^{2} \phi}}
\end{aligned}
$$

and thus

$$
\tilde{k}(t, r)=\rho K(1-\rho) \quad \text { with } \quad \rho=\frac{2 r}{t+r}
$$

and where

$$
K(\alpha)=\int_{0}^{\pi / 2} \frac{d \phi}{\sqrt{1-\alpha^{2} \sin ^{2} \phi}}, \quad|\alpha|<1,
$$

denotes the complete elliptic integral.

Let $\delta>0$ such that $\delta<\operatorname{dist}(\Gamma, \Omega)$. Since $\mathcal{M}_{1}[q](x, \tau)=0$ for $\tau \leq \delta$ we can consider the integral equation $[3.9$ on $[\delta, T]$; that is,

$$
\Phi(x, t)=\int_{\delta}^{t} \tilde{k}(t, r) \mathcal{M}_{1}[q](x, r) d r, \quad t \in[\delta, T] .
$$

Now we note that $\tilde{k}(t, t)=K(0)=\pi / 2$ and $\tilde{k} \in C^{1}(\Delta)$ where $\Delta=\{(t, r) \in$ $\left.[\delta, T]^{2}: r \leq t\right\}$. Therefore, we can transform the equation of the first kind into one of the second kind by differentiating (3.10). This yields

$$
\Phi(x, t)=\frac{\pi}{2} \mathcal{M}_{1}[q](x, t)+\int_{\delta}^{t} \frac{\partial}{\partial t} \tilde{k}(t, r) \mathcal{M}_{1}[q](x, r) d r, \quad t \in[\delta, T] .
$$


Volterra integral equations of the second kind with smooth kernel and nonvanishing diagonal have a unique solution, see, e.g., [13, Section 3.3].

After calculating the spherical mean operator $\mathcal{M}_{1}[q]$, it can be inverted by using standard inversion formulas, which exist for various geometries: For $B=$ $B_{R}^{2}(0) \subset \mathbb{R}^{2}$, analytical reconstruction formulas have been derived by Finch, Haltmeier, Rakesh [6]. For a general domain $\Omega$, Kunyansky reduced in [16] the reconstruction problem to the determination of the eigenvalues $\lambda_{k}$ and normalized eigenfunctions $u_{k},\left\|u_{k}\right\|_{2}=1$, of the Dirichlet Laplacian $-\Delta$ on $\Omega$ with zero boundary conditions. Recently Palamadov [21] presented a general approach leading to reconstruction algorithms for various geometries. For more details see also the Appendix.

Thus the reconstruction algorithm is as follows:

1. Calculate the product $M(x, z, t):=f(z) \check{L}(x, z, t)$ from 3.3 for all $x \in \Gamma, z \in B, t>0$. Compute $\Phi(x, t)$ from 3.8 for all $x \in \Gamma$ and $t>0$.

2. Solve 3.11 for calculating $\mathcal{M}_{1}[q]$.

3. Invert the spherical mean operator.

4. From 3.7 determine $\check{L}$ by differentiation.

5. Finally use $f(z)=M(x, z, t) / \check{L}(x, z, t)$ to reconstruct $f(z)$ for $z \in$ $\Omega$.

\section{Acknowledgements}

The research in this paper was initialized at the Program on Inverse Problems and Applications at MSRI, Berkeley, during the Fall of 2010. It was followed up at the Special Semester at the Newton Institute in 2011.

\section{Appendix}

\section{Notation}

$B_{R}^{n}(x)$ denotes the ball of radius $R$ and center $x$ in $\mathbb{R}^{n}$.

\section{Bessel Functions}

The Bessel functions, Neumann functions and Hankel functions of order zero are defined as

$$
\begin{aligned}
J_{0}(z) & =\frac{1}{2 \pi} \int_{-\pi}^{\pi} e^{\mathrm{i} z \sin (\tau)} d \tau \quad \text { for } z \in \mathbb{C}, \quad \text { and } \\
Y_{0}(z) & =\frac{2}{\pi}[\gamma+\ln (z / 2)] J_{0}(z)-\frac{2}{\pi} \sum_{\ell=0}^{\infty} \frac{a_{\ell+1}}{(\ell !)^{2}}\left(\frac{z}{2}\right)^{2 \ell} \quad \text { for } z \in \mathbb{C} \text { with } \Im \geq 0, \\
H_{0}^{(1)} & =J_{0}+\mathrm{i} Y_{0},
\end{aligned}
$$


where $\gamma$ denotes Euler's constant, $a_{1}=-\gamma$, and $a_{n}=-\gamma+\sum_{\ell=1}^{n-1} \ell^{-1}$ for $n \geq 2$. From this we conclude that $H_{0}^{(1)}(-x)=\overline{H_{0}^{(1)}(x)}$ for $x \in \mathbb{R}$.

\section{The Fourier Transform}

In this paper we use the following notation:

$$
\hat{f}(k):=\mathcal{F} f(k):=\int_{-\infty}^{\infty} f(t) e^{\mathrm{i} k t} d t
$$

denotes the Fourier transform of $f$ and

$$
\check{f}(t):=\mathcal{F}^{-1} f(t):=\frac{1}{2 \pi} \int_{-\infty}^{\infty} f(k) e^{-\mathrm{i} t k} d k,
$$

the inverse Fourier transform. For $f \in L^{1}(\mathbb{R})$ or $f$ from the Schwarz space $\mathcal{S}$ they are defined in the classical sense, for $f \in L^{2}(\mathbb{R})$ they are defined by extension using the Parseval formula, and for tempered distributions they are defined by duality.

\section{Examples:}

$$
\begin{aligned}
\hat{\delta}(k) & =\mathcal{F} \delta(k)=\int_{-\infty}^{\infty} e^{\mathrm{i} k t} \delta(t) d t \equiv e^{\mathrm{i} k 0}=1, \\
\check{\delta}(t) & =\mathcal{F}^{-1} \delta(t)=\frac{1}{2 \pi} \int_{-\infty}^{\infty} e^{-\mathrm{i} k t} \delta(k) d k \equiv \frac{1}{2 \pi} .
\end{aligned}
$$

This shows that

$$
\hat{1}(k)=2 \pi \delta(k) \quad \text { and } \quad \check{1}(t)=\frac{1}{2 \pi} \int_{-\infty}^{\infty} e^{-\mathrm{i} k t} d k=\frac{1}{2 \pi} \delta(t) .
$$

Furthermore, we have (see [20])

$$
\frac{i}{4} H_{0}^{(1)}(k a)=\int_{a}^{\infty} \frac{\exp (i k t)}{2 \pi \sqrt{t^{2}-a^{2}}} d t \quad \text { for } k>0 .
$$

From $H_{0}^{(1)}(-x)=\overline{H_{0}^{(1)}(x)}$ for all $x \in \mathbb{R}$ we conclude that this formula holds for all $k \in \mathbb{R}$ with $k \neq 0$. The right hand side is a Fourier transform. Therefore,

$$
\mathcal{F}^{-1}\left(\frac{i}{4} H_{0}^{(1)}(a \cdot)\right)=\left\{\begin{array}{cl}
\frac{1}{2 \pi \sqrt{t^{2}-a^{2}}}, & t>a, \\
0, & t<a .
\end{array}\right.
$$

\section{Radon Transform}

In $\mathbb{R}^{n}, E(r, \theta)$ denotes the $(n-1)$ - dimensional hyperplane with orientation $\theta \in S^{n-1}$ and distance $r$ from origin.

Let $f: \mathbb{R}^{n} \rightarrow R$ with support in $\Omega$, then the (n-1)-dimensional Radon transform of $f$ is defined by

$$
R_{r, \theta}(f)=R[f](r, \theta)=\int_{E(r, \theta)} f(x) d s(x) .
$$

Thus $R[f]$ is a function from $\mathbb{R} \times S^{n-1}$ into $\mathbb{R}$. 


\section{Spherical Mean Operator}

In $\mathbb{R}^{n}$ the spherical mean operator is defined as follows (see e.g. [5]):

$$
\mathcal{M}_{n-1}[u](x, r):=\frac{1}{\left|S^{n-1}\right|} \int_{S^{n-1}} u(x+r y) d s(y) \text { for } x \in \mathbb{R}^{n}, r>0 .
$$

The spherical mean operator can be written as

$$
\mathcal{M}_{n-1}[u](x, r)=\frac{1}{r^{n-1}\left|S^{n-1}\right|} \int_{\mathbb{R}^{n}} \delta(|x-z|-r) u(z) d z \text { for } x \in \mathbb{R}^{n}, r>0 .
$$

In particular

$$
\begin{aligned}
& \mathcal{M}_{2}[u](x, r)=\frac{1}{4 \pi^{2}} \int_{S^{2}} u(x+r y) d s(y)=\frac{1}{4 \pi^{2} r^{2}} \int_{\mathbb{R}^{3}} \delta(|x-z|-r) u(z) d z \\
& \mathcal{M}_{1}[u](x, r)=\frac{1}{2 \pi} \int_{S^{1}} u(x+r y) d s(y)=\frac{1}{2 \pi r} \int_{\mathbb{R}^{2}} \delta(|x-z|-r) u(z) d z
\end{aligned}
$$

There exist a variety of reconstruction formulas for averages over circular and spherical means:

in $2 \mathrm{D}$

- For averaged data on the sphere of Radius $R$, that is on $\partial \Omega=\partial B_{R}^{2}(0) \subset$ $\mathbb{R}^{2}$, analytical reconstruction formulas have been derived by Finch, Haltmeier, Rakesh [6] and read as follows

$$
f(\xi)=\frac{1}{2 \pi} \Delta_{\xi}\left(\int_{S^{1}} \int_{0}^{2 R} r\left(\mathcal{M}_{1}[f]\right)(\theta, r) \log \left|r^{2}-\right| \xi-\left.\theta\right|^{2} \mid d r d s(\theta)\right)
$$

and

$$
f(\xi)=\frac{1}{2 \pi} \int_{S^{1}} \int_{0}^{2}\left(\partial_{r} r \partial_{r} \mathcal{M}_{1}[f]\right)(\theta, r) \log \left|r^{2}-\right| \xi-\left.\theta\right|^{2} \mid d r d s(\theta) .
$$

- For a general domain $\Omega$, Kunyansky reduced in [16] the reconstruction problem to the determination of the eigenvalues $\lambda_{k}$ and normalized eigenfunctions $u_{k},\left\|u_{k}\right\|_{2}=1$, of the Dirichlet Laplacian $-\Delta$ on $\Omega$ with zero boundary conditions:

$$
\begin{aligned}
& \Delta u_{k}(\xi)+\lambda_{k} u_{k}(\xi)=0, \quad \xi \in \Omega, \\
& u_{k}(\xi)=0, \quad \xi \in \partial \Omega \text {. }
\end{aligned}
$$

Indeed, if $(\xi, \eta) \mapsto G_{\lambda_{k}}(|\xi-\eta|)$ is a free-space rotationally invariant Green's function of the Helmholtz equation and $n(\xi)$ denotes the outer unit normal vector of $\partial \Omega$ at $\xi \in \partial \Omega$, then

$$
f(\xi)=2 \pi \sum_{k=0}^{\infty} \tilde{M}_{k} u_{k}(\xi)
$$

where

$$
\tilde{M}_{k}=\int_{\partial \Omega} \int_{0}^{\infty} r \mathcal{M}_{1}[f](\eta, r) G_{\lambda_{k}}(r)\left\langle\nabla u_{k}(\eta), n(\eta)\right\rangle d r d s(\eta) .
$$


in $3 \mathrm{D}$

We refer to the survey of Finch and Rakesh [8] (see also [7). In this article three reconstruction formulas are documented, which are

$$
\begin{aligned}
& f(x)=-\frac{1}{2 \pi R_{0}} \int_{\partial B_{R_{0}}^{3}} \frac{\left.\partial_{t t}\left(t^{2}\left(\mathcal{M}_{2}[f]\right)\left(x_{0}, t\right)\right)\right|_{t=\left|x-x_{0}\right|}}{\left|x-x_{0}\right|} d s\left(x_{0}\right), \\
& f(x)=-\frac{1}{2 \pi R_{0}} \int_{\partial B_{R_{0}}^{3}} \frac{\left.\left(\partial_{t} t \partial_{t} t\left(\mathcal{M}_{2}[f]\right)\right)\left(x_{0}, t\right)\right|_{t=\left|x-x_{0}\right|}}{\left|x-x_{0}\right|} d s\left(x_{0}\right), \\
& \left.f(x)=-\frac{1}{2 \pi R_{0}} \Delta\left(\int_{\partial B_{R_{0}}^{3}}\left|x-x_{0}\right| \mathcal{M}_{2}[f]\right)\left(x_{0},\left|x-x_{0}\right|\right) d s\left(x_{0}\right)\right) .
\end{aligned}
$$

Here $x \in B_{R_{0}}^{3}$ and $f$ is compactly supported in this set.

\section{References}

[1] M. Agranovsky and P. Kuchment. Uniqueness of reconstruction and an inversion procedure for thermoacoustic and photoacoustic tomography with variable sound speed. Inverse Probl., 23(5):2089-2102, 2007.

[2] G. Bal. Hybrid inverse problems and internal information, 2011. submitted.

[3] G. Bal and G. Uhlmann. Inverse diffusion theory of photoacoustics. Inverse Probl., 26:085010, 2010.

[4] P. Elbau, O. Scherzer, and R. Schulze. Reconstruction formulas for photoacoustic sectional imaging. Preprint on ArXiv arXiv:1109.0841v1, University of Vienna, Austria, 2011.

[5] L. C. Evans. Partial Differential Equations, volume 19 of Graduate Studies in Mathematics. American Mathematical Society, Providence, RI, 1998.

[6] D. Finch, M. Haltmeier, and Rakesh. Inversion of spherical means and the wave equation in even dimensions. SIAM J. Appl. Math., 68(2):392-412, 2007.

[7] D. Finch and Rakesh. The spherical mean value operator with centers on a sphere. Inverse Probl., 23(6):37-49, 2007.

[8] D. Finch and Rakesh. Recovering a function from its spherical mean values in two and three dimensions. In L. V. Wang, editor, Photoacoustic Imaging and Spectroscopy, Optical Science and Engineering, pages 77-87. CRC Press, Boca Raton, FL, 2009.

[9] S. Gratt, K. Passler, R. Nuster and G. Paltauf. Photoacoustic Imaging with a Large, Cylindrical Detector. In Digital Holography and ThreeDimensional Imaging, OSA Technical Digest (CD), paper JMA51, 2011.

[10] S. Gratt, K. Passler, R. Nuster and G. Paltauf. Photoacoustic section imaging with an integrating cylindrical detector. Proc. SPIE 8090, 80900K; doi:10.1117/12.889703, 2011. 
[11] Y. Hristova, P. Kuchment, and L. Nguyen. Reconstruction and time reversal in thermoacoustic tomography in acoustically homogeneous and inhomogeneous media. Inverse Problems, 24(5):055006 (25pp), 2008.

[12] F. John. Plane waves and spherical means applied to partial differential equations. Dover Publications Inc., Mineola, NY, 2004. Reprint of the 1955 original.

[13] R. Kress. Linear integral equations. (2nd edition) Springer, New York, 1999.

[14] P. Kuchment. Mathematics of hybrid imaging - a brief review. 2011.

[15] P. Kuchment and L. A. Kunyansky. Mathematics of thermoacoustic and photoacoustic tomography. European J. Appl. Math., 19:191-224, 2008.

[16] L. A. Kunyansky. A series solution and a fast algorithm for the inversion of the spherical mean radon transform. Inverse Probl., 23(6):S11-S20, 2007.

[17] R. Ma, A. Taruttis, V. Ntziachristos and D. Razansky. Multispectral optoacoustic tomography (MSOT) scanner for whole-body small animal imaging. Optics Express, 17(24):21414-21426, 2009.

[18] S. J. Norton. Reconstruction of a two-dimensional reflecting medium over a circular domain: Exact solution. J. Acoust. Soc. Amer., 67(4):1266-1273, 1980.

[19] S. J. Norton and M. Linzer. Ultrasonic reflectivity imaging in three dimensions: Exact inverse scattering solutions for plane, cylindrical and spherical apertures. IEEE Trans. Biomed. Eng., 28(2):202-220, 1981.

[20] F. Oberhettinger. Tables of Fourier transforms and Fourier transforms of distributions. Springer, Berlin, Heidelberg, New York, 1990.

[21] V. P. Palamodov. A new reconstruction method in integral geometry. arXiv,

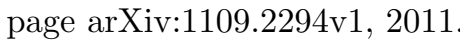

[22] D. Razansky, M. Distel, C. Vinegoni, R. Ma, N. Perrimon, R.W. Köster and V. Ntziachristos. Multispectral opto-acoustic tomography of deep-seated fluorescent proteins in vivo. Nature Photonics, Vol. 3, July 2009:412-417, 2009.

[23] P. Stefanov and G. Uhlmann. Thermoacoustic tomography with variable sound speed. Inverse Probl., 25(7):075011, 16, 2009.

[24] P. Stefanov and G. Uhlmann. Recovery of a source term or a speed with one measurement and applications. preprint, 2011.

[25] M. Xu and L. V. Wang. Exact frequency-domain reconstruction for thermoacoustic tomography-I: Planar geometry. IEEE Trans. Med. Imag., 21:823-828, 2002.

[26] M. Xu and L. V. Wang. Time-domain reconstruction for thermoacoustic tomography in a spherical geometry. IEEE Trans. Med. Imag., 21(7):814822, 2002. 
[27] Y. Xu, D. Feng, and L. V. Wang. Exact frequency-domain reconstrcution for thermoacosutic tomography - i: Planar geometry. IEEE Trans. Med. Imag., 21(7):823-828, 2002.

[28] Y. Xu, M. Xu, and L. V. Wang. Exact frequency-domain reconstruction for thermoacoustic tomography-II: Cylindrical geometry. IEEE Trans. Med. Imag., 21:829-833, 2002. 